\title{
Forebrain glutamatergic neuron-specific Ctcf deletion induces reactive microgliosis and astrogliosis with neuronal loss in adult mouse hippocampus
}

\author{
Ji-Hye Kwak \& Kyungmin Lee \\ Laboratory for Behavioral Neural Circuitry and Physiology, Department of Anatomy, Brain Science \& Engineering Institute, School of \\ Medicine, Kyungpook National University, Daegu 41944, Korea
}

CCCTC-binding factor (CTCF), a zinc finger protein, is a transcription factor and regulator of chromatin structure. Forebrain excitatory neuron-specific CTCF deficiency contributes to inflammation via enhanced transcription of inflammation-related genes in the cortex and hippocampus. However, little is known about the long-term effect of CTCF deficiency on postnatal neurons, astrocytes, or microglia in the hippocampus of adult mice. To address this, we knocked out the Ctcf gene in forebrain glutamatergic neurons (Ctcf cKO) by crossing Ctcf-floxed mice with Camk2a-Cre mice and examined the hippocampi of 7.5-10month-old male mice using immunofluorescence microscopy. We found obvious neuronal cell death and reactive gliosis in the hippocampal cornu ammonis (CA)1 in 7.5-10-month-old cKO mice. Prominent rod-shaped microglia that participate in immune surveillance were observed in the stratum pyramidale and radiatum layer, indicating a potential increase in inflammatory mediators released by hippocampal neurons. Although neuronal loss was not observed in CA3, and dentate gyrus (DG) CTCF depletion induced a significant increase in the number of microglia in the stratum oriens of CA3 and reactive microgliosis and astrogliosis in the molecular layer and hilus of the DG in 7.5-10-month-old cKO mice. These results suggest that long-term Ctcf deletion from forebrain excitatory neurons may contribute to reactive gliosis induced by neuronal damage and consequent neuronal loss in the hippocampal CA1, DG, and CA3 in sequence over 7 months of age. [BMB Reports 2021; 54(6): 317-322]

${ }^{*}$ Corresponding author. Tel: +82-53-420-4803; Fax: +82-53-422-9195; E-mail: irislkm@knu.ac.kr

https://doi.org/10.5483/BMBRep.2021.54.6.265

Received 2 December 2020, Revised 28 December 2020, Accepted 22 January 2021

Keywords: Astrogliosis, Ctcf, Hippocampus, Microgliosis, Neuronal death

\section{INTRODUCTION}

CCCTC-binding factor (CTCF) is a multifunctional nuclear protein with 11 zinc finger DNA-binding motifs in mice (1). Neural CTCF is linked to neurodevelopment, including neural progenitor cell proliferation, differentiation, and survival $(2,3)$. De novo mutations in the human Ctcf gene are associated with phenotypes of various neurodevelopmental disorders, including intellectual disability, microcephaly, and autism $(4,5)$. In addition, neural CTCF plays an important role in cognitive processes with experience-dependent expression of key learning genes, such as Arc and brain-derived neurotrophic factor (6) and CTCFmediated gene expression is required for normal functioning of the hippocampal-cortical networks for recent and long-term memory in adult mice (7).

Furthermore, CTCF is involved in neuroinflammation and neuronal death, which is highly associated with various neurodegenerative and neurological disorders (8). Sams et al. found that mice with CTCF deficiency in postmitotic excitatory forebrain neurons exhibited prominent neuronal apoptosis in the hippocampus and finally early death between 3 and 4 months of age (6). In contrast, in another study, mice with a deletion of Ctcf from the postnatal glutamatergic neurons lived and showed the overexpression of inflammation-related genes leading to abnormal microgliosis, although not neuronal death in the hippocampi of adult mice at 3-4 months of age (9).

Based on these findings, although it has been reported that CTCF regulates age-dependent axonal regeneration and neurodegeneration via the physiological downregulation of Pafah1b1, which is homologous to a gene mutated in lissencephaly (10), the long-term effect of CTCF deficiency in postmitotic excitatory neurons on neuronal death and neurodegeneration remains elusive. Therefore, in this study, we investigated the role of CTCF in cellular senescence or death and explored the neural and glial changes that occur in the hippocampi of 7-month-old mice and older with Ctcf deletions in the forebrain glutamatergic neurons (Ctcf cKO mice). 


\section{RESULTS}

Ctcf gene deficiency in glutamatergic neurons induces neuronal death in the hippocampal CA1, but not in CA3 and dentate gyrus (DG), at 7.5-10 months of age

Loss of CTCF expression in the hippocampi of adult Ctcf cKO mice was confirmed in a previous study (7). Therefore, we examined the effect of Ctcf deficiency in the forebrain glutamatergic neurons on hippocampal cell distribution in mice over 7 months of age. First, immunohistochemistry was performed to label neurons with neuronal nuclear antigen (NeuN), a neuronal marker, in the hippocampus (Fig. 1A). Interestingly, compared to control mice, we observed a visible decrease in the DAPIand NeuN-immunopositive $\left(\mathrm{NeuN}^{+}\right)$signals of the stratum pyramidale (SP) layer in CA1 (Fig. 1A, B), which is the principal cell layer consisting of pyramidal cell somas, in Ctcf cKO mice at 7.5-10 months of age, but not at 4-6 months of age (Supplementary Fig. 1). For quantitative analysis, we measured the width of the SP layer in CA1 and confirmed that it was significantly decreased in Ctcf cKO mice compared with control $(\mathrm{CTL})$ mice (Fig. 1C; CTL, $77.32 \pm 2.55 \mu \mathrm{m} ; \mathrm{CKO}, 55.83 \pm 1.57$ $\mu \mathrm{m} ; \mathrm{t}(8)=7.161, * * * \mathrm{P}=0.000096)$. Next, we counted the $\mathrm{NeuN}^{+}$neuronal number per area in the SP layer of CA1 and found that compared to that in the CTL mice, it was also

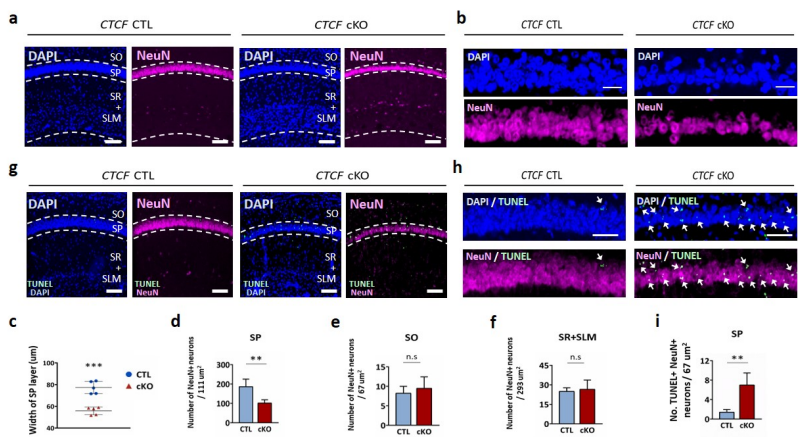

Fig. 1. Ctcf deficiency induces apoptotic cell death in pyramidal neurons of the hippocampal CA1 in CKO mice at 7.5-10 months of age. (A) Immunofluorescence images displaying NeuN (magenta) and DAPI (blue) in the CA1 field of CTL and CKO mice. Two white dashed lines mark the boundary of stratum pyramidale (SP). Scale bars, $100 \mu \mathrm{m}$. (B) High magnification images displaying NeuN (magenta) and DAPI (blue) in the SP layer of CA1. Scale bars, $25 \mu \mathrm{m}$. (C) Width and (D) number of NeuN-immunopositive $\left(\mathrm{NeuN}^{+}\right)$neurons per area of SP. Blue and red dots in (C) represent the data of individual animals. (E, F) Number of $\mathrm{NeuN}^{+}$ neurons per area in the stratum oriens (SO) layer (E) and the region including the stratum radiatum (SR) and stratum lacunosum-moleculare (SLM) layers (SR + SLM, F). (G) Images displaying NeuN (magenta) and TUNEL immunoreactivity (TUNEL ${ }^{+}$, green) in the CA1 field of 7.5-10-month-old control and cKO mice. Scale bars, $100 \mu \mathrm{m}$. $(\mathrm{H})$ High magnification images for $\mathrm{NeuN}^{+}$and $\mathrm{TUNEL}^{+}$neurons in the SP layer of CA1. White arrows indicate $\mathrm{TUNEL}^{+} \mathrm{NeuN}^{+}$neurons. Scale bars, $50 \mu \mathrm{m}$. (I) Number of $\mathrm{TUNEL}^{+} \mathrm{NeuN}^{+}$neurons per area in the SP layer of CA1. n.s., not significant; $* * \mathrm{P}<0.01$, $* * * \mathrm{P}<0.001$. remarkably decreased in the SP layer by approximately $50 \%$ (Fig. 1D; CTL, $186.1 \pm 17.57 / 111 \mu \mathrm{m}^{2} ; \mathrm{cKO}, 101.8 \pm 7.39 / 111$ $\left.\mu \mathrm{m}^{2} ; \mathrm{t}(8)=4.418, * * \mathrm{P}=0.0022\right)$ with a concomitant decrease in the SP layer width in cKO mice at 7.5-10 months of age. However, there was no difference in both the width and $\mathrm{NeuN}^{+}$neuronal number of the SP layer in CA1 of cKO mice at 4-6 months of age (Supplementary Fig. 1). We then counted the number of neurons in the stratum oriens (SO), stratum radiatum (SR), and stratum lacunosum-moleculare (SLM) in CA1, which is also defined as the infrapyramidal, suprapyramidal, and the most superficial region of the hippocampus, respectively. Although the SR and SLM have specific physiological characteristics with different afferents in the hippocampus, exact differentiation of the SR and SLM layers was not possible in the DAPI or NeuN immunofluorescence images. Thus, we counted the neuronal number in the SO layer and whole area including SR and SLM regions (hereafter, SR + SLM layer), and we did not find any differences in the number of $\mathrm{NeuN}^{+}$neurons per area in either SO (Fig. 1E) or SR + SLM layers (Fig. 1F) between genotypes at 7.5-10 months of age.

To confirm whether the decrease in the width and neuronal number of the SP in CA1 of cKO mice was due to neuronal cell death, we investigated the immunoreactivity of TUNEL (terminal deoxynucleotidyl transferase dUTP nick end labeling) staining, which is most widely used to detect cells undergoing apoptotic cell death $(11,12)$, in $\mathrm{NeuN}^{+}$neurons of CA1 (Fig. $1 \mathrm{G})$. As the number of $\mathrm{NeuN}^{+}$neurons was significantly lower in the SP of CA1 in cKO mice than in the SP of CA1 in CTL mice (Fig. 1D), we compared the number of $\mathrm{NeuN}^{+}$neurons showing TUNEL immunoreactivity ( $\mathrm{TUNEL}^{+} \mathrm{NeuN}^{+}$neurons) per area in the SP of CA1 between control and cKO mice at 7.5-10 months of age. We found a remarkably increased number of TUNEL ${ }^{+} \mathrm{NeuN}^{+}$neurons in the SP layer (Fig. $1 \mathrm{H}$, white arrow and Fig. 11; CTL, $1.39 \pm 0.24 / 67 \mu \mathrm{m}^{2}$; cKO, $7.0 \pm$ $\left.1.10 / 67 \mu \mathrm{m}^{2}, \mathrm{t}(9)=-4.933,{ }^{*} \mathrm{P}=0.0011\right)$, suggesting that decreases in the neuronal number and width of the SP were due to apoptotic neuronal death in 7.5-10-month-old Ctcf cKO mice. Next, we examined the neuronal changes in the CA3 and dentate gyrus (DG) of control and cKO mice (Fig. 2A) because Camk2a-cre activity is expressed in both the CA field and DG (9). Intriguingly, we did not observe any differences in $\mathrm{NeuN}^{+}$neuronal numbers in the CA3 area (Fig. 2B-E) and DG (Fig. 2F-I) of control and Ctcf cKO mice.

\section{Ctcf gene deficiency in glutamatergic neurons results in abnormal microgliosis and astrogliosis in the hippocampal CA1 at 7.5-10 months of age}

Based on a previous report demonstrating an increase in inflammation-related gene expression without hippocampal neuronal death in CKO mice at 3-4 months of age (9), we hypothesized that neuroinflammatory responses induced by a long-term deficiency in CTCF expression of glutamatergic neurons would result in neuronal death in the hippocampus of cKO mice over 7 months of age. To address this, we investigated 


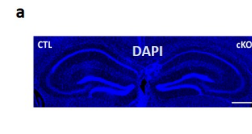

b

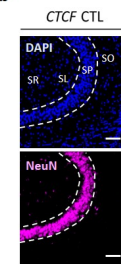

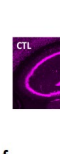
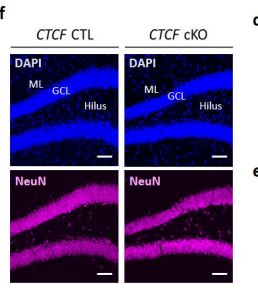
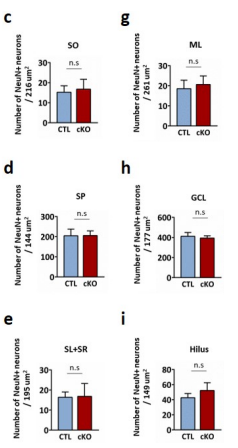

Fig. 2. Neither hippocampal CA3 nor dentate gyrus (DG) show neuronal changes in Ctcf cKO mice at 7.5-10 months of age. (A, B) Immunofluorescence images displaying NeuN (magenta) and DAPI (blue) in the hippocampus (A) and CA3 region (B) of CTL and Ctcf cKO mice. Scale bars, $500 \mu \mathrm{m}$ and $100 \mu \mathrm{m}$ for (A) and (B), respectively. (C-E) Number of $\mathrm{NeuN}^{+}$neurons per area in SO (C), SP (D), and the region consisting of the stratum lucidum (SL) and SR layers (SL + SR, E). (F) Immunofluorescence images displaying NeuN (magenta) and DAPI (blue) in DG. Scale bars, $100 \mu \mathrm{m}$. (G-I) Number of $\mathrm{NeuN}^{+}$neurons per area in the molecular layer $(\mathrm{ML}$, $\mathrm{G})$, granule cell layer $(\mathrm{GCL}, \mathrm{H})$, and hilus (I). n.s., not significant.

glial cells associated with neuroinflammation in the hippocampus of 7.5-10-month-old cKO mice. As expected, reactive microgliosis was clearly observed in all layers of CA1 in Ctcf cKo mice (Fig. $3 \mathrm{~A}, \mathrm{~B})$. Generally, reactive microgliosis during neuroinflammation is highly associated with an increase in the number of ionized calcium-binding adapter protein-1 (lba1) ${ }^{+}$microglia (13). We found that the number of microglia (lba $1^{+}$cells) per area in cKO mice was dramatically increased in the hippocampal $\mathrm{CA} 1$ including the $\mathrm{SO}$ (Fig. 3B, $\mathrm{C} ; \mathrm{t}(4.421)=-23.678, * * * \mathrm{P}=$ 0.0000079), SP (Fig. 3B, D; $t(8)=-13.038, * * * P=0.0000011)$, and SR + SLM (Fig. 3B, E; $\left.\mathrm{t}(8)=-11.902,{ }^{* * * P}=0.0000022\right)$, compared with those in CTL mice. Interestingly, in CKO mice, many of the microglia in the suprapyramidal SR layer of hippocampal CA1 exhibited a bipolar rod shape (Fig. 3B, the lower panel in $\mathrm{CKO}$ ), extending their processes into the SP layer, which showed neuronal loss (Fig. 1A, B). Finally, we examined astrocytes, which play a role in forming a glial scar in damaged nervous tissues (14). As observed in the microglia, we observed dramatic reactive astrogliosis in all layers of the CA1 area in cKO mice compared to control mice (Fig. 3F). In the quantitative analysis, we found increased numbers of glial fibrillary acidic protein (GFAP) ${ }^{+}$astrocytes per area in the whole area of CA1 in Ctcf cKO mice: SO (Fig. 3G, $\mathrm{H} ; \mathrm{t}(5.301)=$ $\left.-3.906,{ }^{*} \mathrm{P}=0.0101\right), \mathrm{SP}$ (Fig. 3G, $\mathrm{l} ; \mathrm{t}(4.826)=-4.039, * \mathrm{P}=$ 0.0106), and SR + SLM layers (Fig. 3G, J; $\mathrm{t}(8)=-4.301,{ }^{*} * \mathrm{P}=$ 0.0026). Thus, our findings showing bipolar rod-shaped microglia (Fig. 3B, lower panels) and hypertrophic astrocytes (Fig. 3G, lower panels) near the SP layer are suggestive of ongoing immune surveillance.
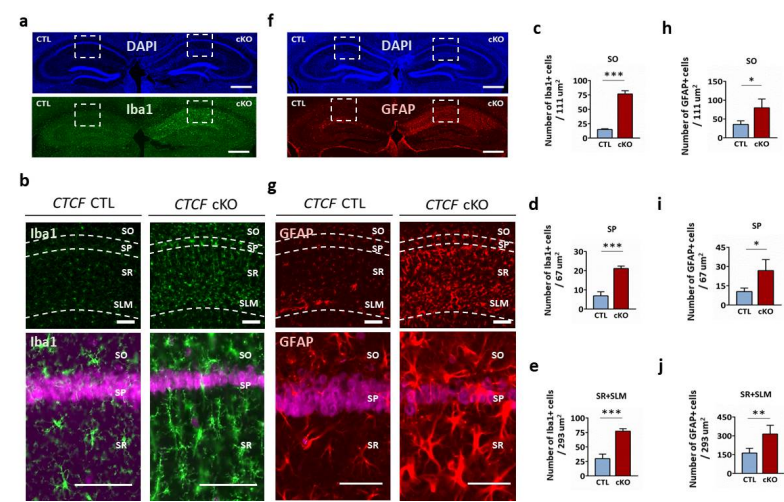

Fig. 3. Ctcf deficiency induces reactive microgliosis with altered microglial morphologies and astrogliosis in CA1. (A) Immunofluorescence images showing Iba1 (green) and DAPI (blue) in the hippocampi of 7.5-10-month-old CTL and Ctcf cKO mice. Scale bars, $500 \mu \mathrm{m}$. (B) Upper panels, images showing Iba1 and DAPI in the CA1 field. Two white dashed lines mark the boundary of SP. Scale bars, $100 \mu \mathrm{m}$. Lower panels, high magnification images showing SO, SP, and SR layer. Note the bipolar rod-shaped microglia extending their processes into the SP (magenta colors) in the CKO mice. Scale bars, $50 \mu \mathrm{m}$. (C-E) Numbers of $\mathrm{Iba}^{+}{ }^{+}$microglia in the SO (C), SP (D), and SR + SLM (E) layers of hippocampal CA1. (F) Immunofluorescence images showing GFAP (red) and DAPI (blue) in the hippocampus. Scale bars, $500 \mu \mathrm{m}$. (G) Upper panels, images of GFAP and DAPI in CA1 field. Scale bars, $100 \mu \mathrm{m}$. Lower panels, high magnification images showing the SO, SP, and SR layers. Scale bars, $50 \mu \mathrm{m}$. (H-J) Numbers of GFAP ${ }^{+}$astrocytes in the SO $(\mathrm{H}), \mathrm{SP}(\mathrm{I})$, and SR + SLM layers $(\mathrm{J})$ of hippocampal CA1. $* P<0.05, * * P<0.01, * * * P<0.001$.

\section{Abnormal microgliosis and astrogliosis occurs prior to neuronal loss in the hippocampal CA3 and DG of Ctcf cKO mice at 7.5-10 months of age}

Next, we examined the changes in glial cells in the hippocampal CA3 and DG of cKO mice, which did not show any neuronal loss compared to the CTL (Fig. 2). Surprisingly, cKO mice exhibited abnormal gliosis in both regions of the hippocampus. In $\mathrm{CA} 3$, only reactive microgliosis was observed in the SO layer of $\mathrm{CKO}$ mice (Fig. 4A, B; $\mathrm{t}(8)=-2.503,{ }^{*} \mathrm{P}=0.0367$ ) and we found neither abnormal microgliosis in the SP, stratum lucidum (SL), and SR layers (Fig. 4A, C, D) nor astrogliosis in $\mathrm{SO}, \mathrm{SP}, \mathrm{SL}$, and SR layers (Fig. 4E-H). Unlike CA3, dramatic reactive microgliosis (Fig. $4 \mathrm{I}$ ) and astrogliosis (Fig. 4M) were visible in the molecular layer (ML) and hilus of DG and quantitative analysis of cell number per area revealed significant increases in $\mathrm{Iba}^{+}$microglia (Fig. 4J for $\mathrm{ML}$ : $\mathrm{t}(8)=-6.634$, $* * * \mathrm{P}=0.00016$; Fig. $4 \mathrm{~L}$ for hilus: $\mathrm{t}(4.175)=-3.068, * \mathrm{P}=$ 0.0353 ) and $\mathrm{GFAP}^{+}$astrocytes (Fig. $4 \mathrm{~N}$ for $\mathrm{ML}: \mathrm{t}(8)=-9.717$, $* * * \mathrm{P}=0.0000105 ;$ Fig. $4 \mathrm{P}$ for hilus: $\mathrm{t}(8)=-2.997, * \mathrm{P}=$ $0.0171)$ in $\mathrm{ML}$ and hilus, but not the granule cell layer (GCL) (Fig. 4K, O) of DG in CKO mice. However, in contrast with CA1, bipolar rod-shaped microglia was not prominently observed in CA3 and DG of cKO mice at the same age (Supplementary Fig. 2). As a result, these findings indicate that 

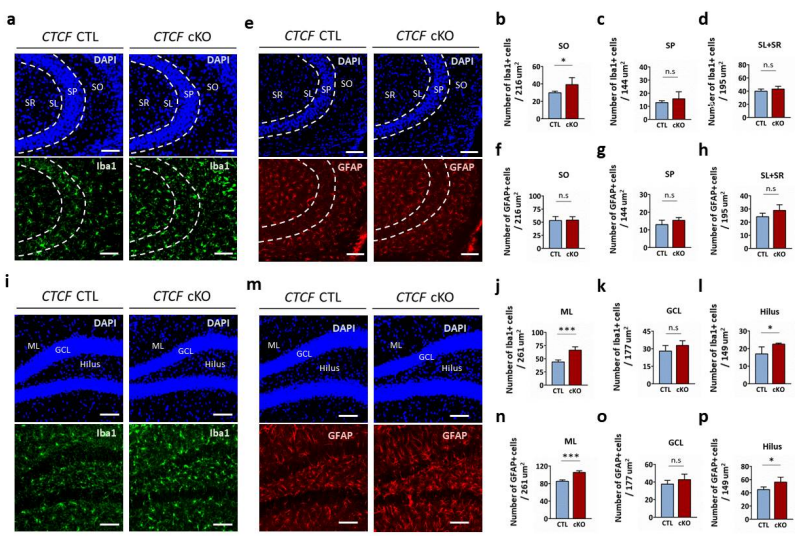

Fig. 4. Ctcf deficiency induces reactive microgliosis and astrogliosis in CA3 and DG over 7.5 months of age. (A, E) Immunofluorescence images showing Iba1 (green, A), GFAP (red, E), and DAPI (blue) in the hippocampal CA3 of CTL and Ctcf cKO mice. Scale bars, $100 \mu \mathrm{m}$. (B-D) Number of $\mathrm{Iba}^{+}$microglia per area significantly increased in the SO (B), but not in the SP (C) and SL + SR (D) layers of CA3 in CKO mice compared with those in the CTL. $(\mathrm{F}-\mathrm{H})$ Number of $\mathrm{GFAP}^{+}$astrocytes per area in the SO (F), SP $(\mathrm{G})$, and $\mathrm{SL}+\mathrm{SR}(\mathrm{H})$ layers of CA3. (I, M) Immunofluorescence images showing Iba1 (green, I), GFAP (red, M), and DAPI (blue) in the DG. Scale bars, $100 \mu \mathrm{m}$. (J-L) The number of $\mathrm{Iba}^{+}$microglia per area significantly increased in the $M L(J)$ and hilus (L), but not in the GCL $(K)$ of DG in cKO mice. (N-P) The number of GFAP ${ }^{+}$ astrocytes per area increased in the $M L(N)$ and hilus $(P)$, but not in GCL (O) of DG in CKO mice compared with those in the CTL. n.s., not significant; $* P<0.05, * * * P<0.001$.

neuroinflammatory responses to potent neuronal damage induced by Ctcf gene deficiency in glutamatergic neurons occur much earlier and more intensively in DG than CA3.

\section{DISCUSSION}

In a previous report, 3-4-month-old Ctcf cKO mice demonstrated abnormal microgliosis without astrogliosis and neuronal cell death in the CA1 of the hippocampus (9). However, in the current study, we propose that a prolonged CTCF deficiency in postmitotic glutamatergic neurons induces neuroinflammation in parallel with reactive microgliosis as well as astrogliosis and consequent excitatory neuronal loss specifically in the CA1 of the hippocampus over 7 months of age because we did not find any neuronal loss in the CA1 of CKO mice at 4-6 months of age. On the basis of the previous data showing a significant decrease in dendritic spine density of pyramidal neurons and remarkable increase in inflammation-related gene expression such as toll-like receptor 2 as an activator of microglia within the CA1 of 3-4-month-old cKO mice (9), CTCF deletion-induced neuronal damage and consequent neuroinflammatory responses with inflammatory gene expression may induce reactive gliosis prior to neuronal loss in cKO mice over 7 months of age.

Interestingly, we observed abnormal microgliosis and astrogliosis in the DG and only microgliosis in CA3 without neuronal loss in 7.5-10-month-old cKO mice. Based on these findings, we speculate that neuronal damage induced by the depletion of CTCF causes abnormal gliosis of microglia, followed by reactive astrogliosis and neuronal death in hippocampal CA1 at 3-10 months of age. Furthermore, neuronal damage and neuroinflammation occur first in the hippocampal CA1 and next in the DG, and finally in the CA3 of Ctcf cKO mice. We speculate that the relatively high resistance of DG and CA3 against CTCF deficiency-induced neuronal damage may be associated with neurogenesis-related capability in the DG and recurrent excitatory circuit in the CA3 because synaptic activity can have an influence on neuronal survival. However, we cannot rule out the different efficiency of CTCF deletion caused by the different expression of Cre recombinase in the hippocampal subfields of adult Camk2a-Cre mice and further studies are needed to elucidate an exact mechanism underlying different phenotypes between hippocampal subregions.

Neuronal death following sequential microgliosis and astrogliosis was also observed in the anterior cingulate cortex (ACC) of CKO mice. Indeed, our previous study on the ACC demonstrated apoptotic cell death of cortical neurons in CKO mice over 5 months of age, subsequent to age-dependent aggravation in reactive gliosis at 4 months of age (15). However, in the present study, we found that hippocampal neuronal damage and death induced by CTCF deficiency occur later than cortical neuronal death of the ACC and we assume that it might attribute to enrichment of neurogenesis-related genes in the hippocampus.

We also found microglia with a bipolar rod shape, which is considered a transitional stage between the ramified microglia for immune surveillance (16) and amoeboid microglia for phagocytosis (17), specifically in the SR layer of the CA1, but neither CA3 nor DG, in 7.5-10-month-old cKO mice. In humans, the presence of rod-shaped microglia in the hippocampus is directly associated with age-dependent increases in senescence and neurodegeneration-related pathologies, such as dementia and senile plaque formation in the brain (18). In addition, CTCF itself as an epigenetic factor has known to play a pivotal role in transcriptional regulation of huntingtin (19), TMEM106B (20), and amyloid precursor protein (21), which are highly associated with neurodegeneration. Therefore, more detailed studies to investigate direct pathophysiological evidence of neuronal senescence and neurodegeneration in the hippocampus of $\mathrm{CKO}$ mice will provide insight into the age-dependent role of CTCF in the neuroinflammation processes of neurodegenerative diseases in the future.

\section{MATERIALS AND METHODS}

\section{Animals}

Ctcf ${ }^{\text {doxplloxp }}$ mice and Camk $2 \alpha-C r e$ mice were described previously by Heath et al. (22) and by Madisen et al. (23), respectively. $\mathrm{Ctcf}^{\text {foxp }}$;Camk2 $\alpha-\mathrm{Cre}^{+}$mice were described elsewhere in Kim et al. (7) and Kwak et al. (15). Littermates without the Cre 
transgene or the floxed Ctcf gene were used as controls (CTL). The Animal Care and Use Committee of Kyungpook National University approved the animal protocols. The following are the number of animals and brain slices for immunohistochemistry: CTL 5 (brain slices 10), and Ctcf cKO 5 (brain slices 10) for NeuN-, GFAP-, and Iba1-expressing cells and TUNEL ${ }^{+}$ cells.

\section{Immunohistochemistry, TUNEL staining, and imaging}

Brain sample preparation and immunohistochemistry were performed as previously described (24). After tissue preparation (coronal slices, $40 \mu \mathrm{m}$ thickness), the brain sections were incubated with the following primary antibodies at 1:1,000 dilution: mouse anti-NeuN (MAB377; Merck Millipore, Burlington, MA, USA), rabbit anti-GFAP (Z0334; Dako, Glostrup, Denmark) overnight at $4^{\circ} \mathrm{C}$, and goat anti-ionized calcium-binding adapter protein-1 (lba1) (NB100-1028; Novus Biologicals, Littleton, CO, USA) for 2 days at $4^{\circ} \mathrm{C}$. The sections were then incubated with the following secondary antibodies at 1:200 dilutions for $3 \mathrm{~h}$ at room temperature: Cy5-conjugated anti-mouse (for anti-NeuN), Alexa Fluor 546 donkey anti-rabbit IgG (for anti-GFAP), and Alexa Fluor 488 donkey anti-goat IgG (for anti-lba1). For TUNEL assay, sections were labeled with an in situ cell death detection kit following the manufacturer's instructions (Roche Applied Science, no. 11684795910 ) at $37^{\circ} \mathrm{C}$ for $1 \mathrm{~h} 30 \mathrm{~min}$. $\mathrm{TUNEL}^{+}$cells were indicated by green fluorescence. After incubation with DAPI at 1:20,000 dilutions, images of the immunostained sections were acquired with a Zeiss (Thornwood, NY, USA) confocal microscope and an LSM5 EXCITER, version 4.2 (25). Fluorescence images were analyzed using ImageJ (NIH, Bethesda, MD, USA) and MATLAB (Mathworks, Natick, MA, USA).

\section{Experimental design and statistical analysis}

All experiments were performed in a blinded fashion, with the minimum number of mice required to achieve statistical validity. Data are shown as the mean \pm standard error of the mean. For each variable, two groups were compared using a two-tailed $t$-test if the data were normally distributed. Statistical significance was set at $\mathrm{P}<0.05$.

\section{ACKNOWLEDGEMENTS}

This study was supported by the National Research Foundation (NRF) of Korea grants funded by the Korean government (MSIP) [NRF- 2019R1F1A1063932] to K.L.

\section{CONFLICTS OF INTEREST}

The authors have no conflicting interests.

\section{REFERENCES}

1. Ong CT and Corces VG (2014) CTCF: an architectural protein bridging genome topology and function. Nat Rev Genet 15, 234-246

2. Moore JM, Rabaia NA, Smith LE et al (2012) Loss of maternal CTCF is associated with peri-implantation lethality of Ctcf null embryos. PLoS One 7, e34915

3. Watson LA, Wang X, Elbert A, Kernohan KD, Galjart N and Berube NG (2014) Dual effect of CTCF loss on neuroprogenitor differentiation and survival. J Neurosci 34, 2860-2870

4. Gregor A, Oti M, Kouwenhoven EN et al (2013) De novo mutations in the genome organizer CTCF cause intellectual disability. Am J Hum Genet 93, 124-131

5. Iossifov I, O'Roak BJ, Sanders SJ et al (2014) The contribution of de novo coding mutations to autism spectrum disorder. Nature 515, 216-221

6. Sams DS, Nardone S, Getselter D et al (2016) Neuronal CTCF is necessary for basal and experience-dependent gene regulation, memory formation, and genomic structure of BDNF and Arc. Cell Rep 17, 2418-2430

7. Kim S, Yu NK, Shim KW et al (2018) Remote memory and cortical synaptic plasticity require neuronal CCCTC-binding factor (CTCF). J Neurosci 38, 5042-5052

8. Gorman AM (2008) Neuronal cell death in neurodegenerative diseases: recurring themes around protein handling. J Cell Mol Med 12, 2263-2280

9. McGill BE, Barve RA, Maloney SE et al (2018) Abnormal microglia and enhanced inflammation-related gene transcription in mice with conditional deletion of Ctcf in Camk2aCre-expressing neurons. J Neurosci 38, 200-219

10. Kumamoto $K$, Iguchi $T$, Ishida $R$, Uemura $T$, Sato $M$ and Hirotsune S (2017) Developmental downregulation of LIS1 expression limits axonal extension and allows axon pruning. Biol Open 6, 1041-1055

11. Gavrieli Y, Sherman Y and Ben-Sasson SA (1992) Identification of programmed cell death in situ via specific labeling of nuclear DNA fragmentation. J Cell Biol 119, 493-501

12. Nagata S (2000) Apoptotic DNA fragmentation. Exp Cell Res 256, 12-18

13. Lana D, Ugolini F, Wenk GL, Giovannini MG, ZecchiOrlandini S and Nosi D (2019) Microglial distribution, branching, and clearance activity in aged rat hippocampus are affected by astrocyte meshwork integrity: evidence of a novel cell-cell interglial interaction. FASEB J 33, 4007-4020

14. Wanner IB, Anderson MA, Song B et al (2013) Glial scar borders are formed by newly proliferated, elongated astrocytes that interact to corral inflammatory and fibrotic cells via STAT3-dependent mechanisms after spinal cord injury. J Neurosci 33, 12870-12886

15. Kwak JH, Kim S, Yu NK et al (2020) Loss of the neuronal genome organizer and transcription factor CTCF induces neuronal death and reactive gliosis in the anterior cingulate cortex. Genes Brain Behav 20, e12701

16. Au NPB and Ma CHE (2017) Recent advances in the study of bipolar/rod-shaped microglia and their roles in neurodegeneration. Front Aging Neurosci 9, 128

17. Jonas RA, Yuan TF, Liang YX, Jonas JB, Tay DK and Ellis-Behnke RG (2012) The spider effect: morphological and orienting classification of microglia in response to stimuli in vivo. PLoS One 7, e30763 
18. Bachstetter AD, Van Eldik LJ, Schmitt FA et al (2015) Disease-related microglia heterogeneity in the hippocampus of Alzheimer's disease, dementia with Lewy bodies, and hippocampal sclerosis of aging. Acta Neuropathol Commun 3, 32

19. De Souza RA, Islam SA, McEwen LM et al (2016) DNA methylation profiling in human Huntington's disease brain. Hum Mol Genet 25, 2013-2030

20. Gallagher MD, Posavi M, Huang P et al (2017) A dementia-associated risk variant near TMEM106B alters chromatin architecture and gene expression. Am J Hum Genet 101, 643-663

21. Yang Y, Quitschke WW, Vostrov AA and Brewer GJ (1999) CTCF is essential for up-regulating expression from the amyloid precursor protein promoter during differentiation of primary hippocampal neurons. J Neurochem 73, 22862298

22. Heath H, Ribeiro de Almeida C, Sleutels F et al (2008) CTCF regulates cell cycle progression of alphabeta T cells in the thymus. EMBO J 27, 2839-2850

23. Madisen L, Zwingman TA, Sunkin SM et al (2010) A robust and high-throughput Cre reporting and characterization system for the whole mouse brain. Nat Neurosci 13, 133-140

24. Seo H and Lee K (2019) Cell-specific expression of Epac2 in the subventricular and subgranular zones. Mol Brain 12, 113

25. Park SW, Jun YW, Choi HE et al (2019) Deciphering the molecular mechanisms underlying the plasma membrane targeting of PRMT8. BMB Rep 52, 601-606 